\title{
Riemannian Manifolds for which a Power of the Radius is k-tharmonic
}

\author{
R. SCHIMMTríg
}

Es, sei $\sigma=\sigma(x, y)$ die Syngesche Funktion einer Riemannschen Mannigfaltigkeit $(M, g)$ belièbiger Signatur. Wir betrachten dic Bedingung, daß eine bestimmte Potenz von $\sigma$ oder der Logarithmus von $\sigma k$-harmonisch ist. Dann erweist sich $(M L, g)$ in vielen Fällen als flach. Be; stimmte Klassen nicht-flacher Mannigfaltigkeiten können durch eine Bedingung vom genannten Typ charakterisiert werden.

Пусть $\sigma=\sigma(x, y)-$ функция Синга Риманого многообразия $(M, g)$ произвольной сигиатуры. Рассматривается условие, -что некоторая степень $\sigma$ или -погарифм $\sigma$ является $k$-гармдонической функцией. Тогда в многих случаях $(M, g)$, оказывается плоским: Некоторые классы неплоских многообразий могут быть охарактеризованы. условием названиого типа.

Let $\sigma=\sigma(x, y)$ denote Synge's function of a Riemannian manifold $(\ddot{M}, g)$ of any signature and consider the condition that some power of $\sigma$ or the logarithm of $\sigma$ is $k$-harmonic. Then in many cases $(M, g)$ turns out to be flat. Certain classes of non-flat manifolds can be characterized just by a condition of the aforesaid type.

\section{Introduction}

For points $x, y$ of a smooth $n$-dimensional properly Riemannian manifold $(M, g)$ which are not too far from each other the geodesic distance $r=r(x, y)$ is defined. Fór fixed $y$ and variable $x$ we call $r=r(x, y)$ shortly the radius. Recently the problem

$$
\Delta^{k} r^{2 t}=0
$$

has been posed, i.e. the condition that some power of the radius is $k$-harmonic $[2-4,6,7]$. Here $k$ denotes a positive integer, $l$ a real number, and

$$
\begin{aligned}
& \Delta:=g^{\alpha \beta} \nabla_{\alpha} \nabla_{\beta}=\text { Laplace operátor to } g, \\
& \nabla=d x^{\alpha} \nabla_{\alpha}: \doteq \text { Levi-Civita derivative to } g, \\
& g=g_{\alpha \beta} \dot{d} x^{\alpha} d x^{\beta}=\text { Riemannian metric, } \\
& \left(g^{\alpha \beta}\right):=\left(g_{\alpha \beta}\right)^{-1} .
\end{aligned}
$$

One motivation for the problem comes from the fact that certain classes of manifolds can be characterized just by a condition (1), namely the simply harmonic spaces with $n \geqq 3$ by $k=1,2 l=2-n$, and the 3 -dimensional spaces of constant curvature by $k=2,2 l=1$. On the one hand it is an aim to find further such characterizations, on the other hand it is to be expected that for many combinations $(k, l, n)$ the condition (1) implies local flatness. For instance, [4] is devoted to the (still open) conjecture that this should be the case for $k=2,2 l=2-n<0$. There 
is also another motivation: for $2\left(k^{\prime}-l\right)=n, l<0$ from (1) there follows that $\Delta^{k-1} r^{2 l}$ is an elementary solution of the Laplace equation. In general it is very difficult to calculate an elementary solution, in our special case we have an explicit expression for it.

The present paper will introduce some new aspects:

I. The Riemannian metric $g$ may be of any signature (properly or, pseudo-Riemannian). The usual definition of the geodesic distance fails then; instead Synge's two-point function $\sigma=\sigma(x ; y)$ is to be taken as a primary quantity and

$$
r:=2|\sigma|^{1 / 2}, \quad e:=\operatorname{sign} \sigma
$$

as secondary quantities. It will turn out that "results are available especially for lorentzian $g$, i.e. for a signature $(+-\cdots-)$ or $(-+\cdots+)$.

II. We will study the problem

$$
\dot{\Delta}^{k}(\log r)=0
$$

too. It is a natural completion of. (1) because

$$
\lim _{i \rightarrow 0} \Delta^{k} r^{2 l} / 2 l=\Delta^{k}(\log r)
$$

III.' There are no upper bounds for the numbers $k, l, n$ in our results. Contrarily, the literature until now concentrated to small values of $k$. $J$. EICHHORN [:7] studied also general $k$ for definite $g$.

We always consider $y \in M$ as fixed and $x \in M$ as variable; all differential operations refer to the point $x$. Let $N(y)$ denote a normal neighbourhood of $y \in M$; in it $\sigma=\sigma(x, y)$ is defined. Let further

$$
N(y)^{-}:=\{x \in N(y) \mid \sigma(x, y)+0\} .
$$

Now we make precise the

Problems: Search for quadrupels $(M, g, k, l)$ consisting in

- a manifold $M$ of class $C^{\infty}$ and of dimension $n=: 2 m+2 \geqq 2$,

- a Riemunnian metric $g$ over $M$ of class $C^{\infty}$ and of any signature,

- a positive integer $k$,

- a real number.l,

such that for each $y \in M$

$$
\Delta^{k}|\sigma|^{l}=0 \text { in } N(y)^{-} \text {. }
$$

This problem we will abbreviate by $\Delta^{k} \sigma^{l}=0$.

Search also for tripels $(M, g, k)$ such that analogously for each $y \in M$

$$
\Delta^{\dot{k}}(\log |\sigma|)^{\prime}=0
$$

This problem we will abbreviate by $\Delta^{k} \log \dot{\sigma}=0$.

'Here we present a selection of our results:

1. If $\Delta^{k} \sigma^{l}=0$ then one of the numbers $l$ or $l+m$ is an integer between 0 and $k-1$ (inclusively).

Examples:

1.1. If $\Delta \sigma^{l}=0$ then $l=0$ or $l=-m$.

1.2. If $\Delta^{2} \sigma^{l}=0$ then $l$ is one of the numbers $0,1,-m, 1 \stackrel{\prime}{\prime}$.

2. If $\Delta^{k} \log \sigma=0$ then $n$ is an even number and $n \leqq 2 k$. 
Examples:

2.1. If $\Delta \log \sigma=0$ then $n=2$.

2.2. If $L^{2} \log \sigma=0$ then $n=2$ or $n=4$.

3. From $\Delta^{k} \sigma^{l}=0$ there follows local flatness if one of the following additional assumptions is fulfilled:

3.1. $k=2, l=1, n \leqq 5, g$ definite.

3.2. $k=2, l=-m, n \geqq \widetilde{5}, g$ lorentzian.

3.3. $k=2, l=1-m, n \leqq 5$, g.definite or lorentzian.

3.4. $k=3, l=1, n \leqq 5, g$ definite, $R=0$.

3.5. $k \geqq 2, l=k-1, n=2$.

3.6. $4 \leqq k \leqq m, l+m=k-1, n$ even, g lorentzian.

3.7: $4 \leqq k \neq n-1, l+m=k-1, n$ odd, g lorentzian.

3.8. $4 \leqq k \leqq m+1, l+m=k-2, n$ even, $g$ lorentzian, $R=0$;

3.9. $k \geqq 4, i+m=k-2, n$ odd, g lorentzian, $R=0$.

4. From $\Delta^{k} \log \sigma=0$ there follows local flatness if one of the following additional assumptions is fulfilled:

4.1. $k \leqq 2, n=2$.

4.2. $2 \leqq k \leqq m+1, g$ lorentzian.

4.3. $3 \leqq k \leqq m+2, g$ definite.

4.4. $k=m+1, n \geqq 4, g$ definite or lorentzian.

5. There exist non-flat manifolds of any dimension $n \geqq 4$ (namely simply harmonic manifolds) satisfying for any $k \Delta^{k} \sigma^{k-1}=0$ and $\Delta^{k} \sigma^{k-m-1}=0$.

6. There exist non-flat manifolds of any even dimension $n \geqq 4$ (namely simply harmonic manifolds) satisfying $\Delta^{m+1} \log \sigma^{-}=0$.

7. There exist non-flat manifolds of any dimension $n \geqq 3$ and of uny non-definite signature. (namely generalizations of the plane gravitational waves) satisfying $\Delta^{2} \sigma=0$.

8. The 3-dimensional manifolds of constant curvature (of any signature) are characterized by $\Delta^{2}|\sigma|^{1 / 2}=0$.

9. If $\Delta^{k}|\sigma|^{k-m-1}=0$ and $\left(\begin{array}{c}m \\ k\end{array}\right)+0$, then $\Delta^{k-1}|\sigma|^{k-m-1}$ is an elementary solution of the Laplace equation. This is logarithm-free for even $n$.

10. If $\Delta^{m+1}(\log |\sigma|)=0$ and $n \geqq 4$ is even then $\Delta^{m}(\log .|\sigma|)$ is a logarithm-free elementary solution of the Laplace equation.

Let us shortly review results in the literature on the problem $\Delta^{k} \sigma^{l}=0$ for the properly Riemannian case: R. CADDEO [2] proved that $\Delta^{2} r=0$ if and only if $n=1$ or if $(M, g)$ is a 3 -dimenisional manifold of constant curvature. R. CADDEo and P. Matzeu [3] showed that $\Delta^{2} r^{4-n}=0$ if and only if $(M, g)$ is locally flat or is a 3-dimensional manifold of constant curvature. In the papers $[3,4]$ the problems $\Delta^{2} r^{2}=0$ and $\Delta^{2} r^{2-n}=0$ are considered. Necessary conditions are derived; under certain additional assumptions these conditions imply local flatness. Especially, R. CADDEo and L. VANHECKE [4] have shown that an odd-dimensional $(M, g)$ satisfying $\Delta^{2} r^{2-n}=0$ is a harmonic manifold. J. EICHHORN [6] proved that a harmonic manifold satisfying $\Delta^{2} r^{2}=0$ is simply harmonic, which implies local flatness. In [7] he initiated the consideration of arbitrary large $k$ and became able to discuss a number of concrete examples.

The three problems to characterize all Rienannian manifolds $(M, g)$ which

- satisfy $\Delta^{k} \sigma^{l}=0$ or $\Delta^{k} \log \sigma=0$,

- are harmonic ones,

- admit a logarithm-free elementary solution of the Laplace equation 
respectively have much in common. They are of comparable difficulty and none of the problems is solved yet. Possibly, future progress in one of the problems will influence the other two problems.

Our main tool will be a version of the "method of coincidence limits", which has been initiated-by J.L. SYNGE [13], combined with the calculus of synmetric differential forms. It is to be noted that the method is not yet exhausted by the results which are presented here.

I wish to express my thanks to A: GRAY and to J. EICHHORN for suggesting the problem and to the authors of $[2-4]$ for giving me insight into their work.

\section{\$ 1. Symmetric differential forms. The two-point.functions $\sigma$ and $\mu$}

A symmetric differential form of degree $p$

$$
u=u_{p}=u_{\alpha_{1} \alpha_{1} \ldots \alpha_{p}} d x^{\alpha_{1}} d x^{\alpha_{1}} \ldots d x^{a_{p}}
$$

is a special notation for a symmetric covariant tensor field of degree $\dot{p}$ with local components $u_{\alpha_{1} \alpha_{2} \ldots \alpha_{p}}$ with respect to a local chart $x \mapsto\left(x^{\alpha}\right)=\left(x^{1}, x^{2}, \ldots, x^{n}\right.$.). Apart from the usua! tensorial operations there are specific operations for symmetric forms:

1. Symmetric product of a.p-form $u_{p}$ and a $q$-form $v_{q}$

$$
u_{p} v_{q}:=u_{\alpha_{1} \ldots \alpha_{p}} v_{\beta_{1} \ldots \beta_{q}} d x^{\alpha_{1}} \ldots d x^{\alpha_{\nu}} d x^{\beta_{2}} \ldots d x^{\beta_{q}} .
$$

2. Trace $=\operatorname{tr}$ with respect to the metric $g$

$$
\begin{aligned}
& \operatorname{tr} u_{p}:=g^{\alpha \beta} u_{\alpha \beta \alpha_{z} \ldots \alpha_{p}} d x^{\alpha_{3}} \ldots d x^{\alpha_{p}} \text { for } p \geqq 3, \\
& \operatorname{tr} u_{0}:=0, \quad \operatorname{tr} u_{1}:=0, \quad \operatorname{tr} u_{2}:=g^{\alpha \beta} u_{\alpha \beta} .
\end{aligned}
$$

3. Trace-free part $-u_{p}$ of $u_{p}$ with respect to $g$. For $p \leqq 4$ one has

$$
\begin{aligned}
& -u_{0}=u_{0}, \quad-u_{1}=u_{1}, \quad-u_{2}=u_{2}-\frac{1}{n} g \cdot \operatorname{tr} u_{2}, \\
& (n+2)\left(-u_{3}-u_{3}\right)=-3 g \cdot \operatorname{tr} u_{3}, \\
& (n+2)(n+4)\left(\Sigma u_{4}-u_{4}\right)=-6(n+2) g \cdot \operatorname{tr} u_{4}+3 g^{2} \cdot \operatorname{tr}^{2} u_{4} .
\end{aligned}
$$

4. Symmetric differential $d$ built by means of $\nabla$

$$
d u_{p}:=\nabla_{\alpha} u_{\alpha_{1} \ldots \alpha_{p}} d x^{\alpha} d x^{\alpha_{1}} \ldots d x^{\alpha}{ }^{\alpha} .
$$

Lateron we will make use of the formula

$$
\begin{aligned}
& (p+2)(p+1) \operatorname{tr}^{k}\left(g u_{p}\right) \\
& =4 k(m+p-k+2) \operatorname{tr}^{k-1} u_{p}+(p+2-2 k)(p+1-2 k) g \operatorname{tr}^{k} u_{p}
\end{aligned}
$$

We choose the notations and conventions

$$
\begin{aligned}
& \text { Riem }=R_{\alpha \beta \mu \nu}\left(d x^{\alpha} \wedge d x^{\beta}\right)\left(d x^{\mu} \wedge d x^{\nu}\right)=\text { curvature tensor, } \\
& \left(\nabla_{\alpha} \nabla_{\beta}-\nabla_{\beta} \nabla_{\alpha}\right) v^{\mu}=: R_{\alpha \beta}{ }^{\mu} \cdot v^{\nu} \text { for vector fields } v=v^{\alpha} \partial_{\alpha}, \\
& R i c=R_{\alpha \beta} d x^{\alpha} d x^{\beta}=\text { Ricci tensor }:=g^{\mu \nu} R_{\alpha \mu \nu \beta} d x^{\alpha} d x^{\beta}, \\
& R=\text { scalar curvature }:=g^{\alpha \beta} R_{\alpha \beta}, \\
& W e y l=W_{\alpha \beta \mu \nu}\left(d x^{\alpha} \wedge d x^{\beta}\right)\left(d x^{\mu} \cdot \wedge d x^{\prime}\right)=\text { conformal curvature tensor } .
\end{aligned}
$$


For quadratic expressions in the curvature we adopt special notations:

$$
\begin{aligned}
& |R i c|^{2}:=R_{\alpha \beta} R^{\alpha \beta}, \quad|R i e m|^{2}:=R_{\alpha \beta \mu \nu} R^{\alpha \beta \mu r}, \\
& (R i e m)^{2}:=R_{\alpha \lambda_{\ell} \beta} R_{\mu}{ }^{\lambda \rho}, d x^{\alpha} \cdot d x^{\beta} d x^{\mu} d x^{\gamma} .
\end{aligned}
$$

Proposition 1.1: A lorentzian metrï $g$ with $n \geqq 4$ and $-(\text { Weyl })^{2}=0$ is conformally flat.

A proof follows from a close inspection of the arguments which are given by A. LICHNEROWICZ and A. G. WALKER in [10], and also slightly more explicit by H. S. Ruse, A. G. WALKER and T. J. Wrimione in section 2.7 of their book [11]. Thus the proposition may be considered as known, though to our. knowledge not explicitly stated before. Note that similar - but not identical - arguments occur in the context of the Bel-Robinson tensor in general relativity [1, 12]

Proposition 1.2: A lorentzian metric $g$ with $n \geqq 3$ and - Ric $=0$ and $-(\text { Riem })^{2}=0$ is of constant curvature.

Proof: For $\dot{n}=3$ the assertion follows from ${ }^{-}$Ric $=0$. For $n \geqq 4$ we apply Proposition 1.1. Namely, from $-R i c=0$ there follows

$$
(\dot{R i e m})^{2}=(W e y l)^{2}+(n-1) K^{2} g^{2} \text { with } n(n-1) \dot{K}:=R,
$$

and as a conclusion $-(\text { Riem })^{2}=-(\text { Weyl })^{2}$

Definition 1.1: Let $N(y)$ be a normál neighbourhood of $y \in M$, i.e. the exponential map with origin $y$

$$
\exp _{y}: x^{*} \mapsto x
$$

is a diffeomorphism out of the tangential space of $y$ onto $N(y)$ or, equivalently, $N(y)$. is the domain of a normal coordinate system

$$
x \mapsto x^{*} \mapsto\left(x^{* \alpha}\right) \equiv\left(x^{* 1}, x^{* 2}, \ldots, x^{* n}\right)
$$

The quantity

$$
\begin{aligned}
& \sigma=\sigma(x, y):=\frac{1}{2} g(y)\left(\exp _{\nu}{ }^{-1} x, \exp _{\nu}{ }^{-1} x\right) \\
& \quad=\frac{1}{2} g(y)\left(x^{*}, x^{*}\right)=\frac{1}{2} g_{\alpha \beta}(y) x^{* \alpha} x^{* \beta} \text { for } x \in N(y)
\end{aligned}
$$

is called Synge's two-point function. From $\sigma$ there are derived

$$
e=e(x, y):=\operatorname{sign} \sigma(x, y), \ldots, \mu=\mu(x, y):=\frac{1}{2}(\Delta \sigma-n) .
$$

The two-point scalar fields $\sigma$ and $\mu$ are ingredients of the "methód of coincidence limits" which is due to J. L. SYNGE [13].- The limit for $x \rightarrow y$, if existing, of a twopoint quantity is called coincidence limit. The equality of the coincidence limits is an equivalence relation of two-point quantities and will be denoted by $\doteq$. One-point quantities and constants may be looked upon as special two-point quantities.

Let us recall some properties of $\sigma$ and $\mu$ following $[9,13,11,12]$ :

Symmétry: $\sigma(x, y)=\sigma(y, x)$.

Coincidence limits:

$$
\begin{aligned}
& \sigma \doteq 0, \quad \ddot{\nabla_{\alpha}} \sigma \stackrel{0}{0}, \quad \nabla_{a} \nabla_{\beta} \sigma \stackrel{\circ}{=} g_{\alpha \beta}, \\
& \nabla_{\alpha} \nabla_{\beta} \nabla_{\gamma} \sigma \doteq 0, \quad-3 \nabla_{\alpha} \nabla_{\beta} \nabla_{\gamma} \nabla_{\delta} \sigma \doteq R_{y \alpha \beta \delta}+R_{\delta \alpha \beta \gamma} \text {. }
\end{aligned}
$$


Differential equation: $g^{\alpha \beta} \nabla_{\alpha} \sigma \nabla_{\beta} \sigma=2 \sigma$.

Ledger's formula:

$$
-(p+1) d^{p} \sigma_{\alpha \beta} \stackrel{\circ}{=} p(p-1) d^{p-2}\left(R_{\gamma \alpha \beta \delta} d x^{\gamma} d x^{\delta}\right)+\sum_{q=2}^{p-2} \cdot\left(\begin{array}{l}
p \\
q
\end{array}\right) d^{q} \sigma_{\alpha, \gamma} d^{p-q} \sigma_{\beta}{ }^{\gamma}
$$

for $p \geqq 2$, where the sum for $p=2$ and for $p=3$ is to be taken as zero and where we abbreviate. $\sigma_{\alpha}:=\nabla_{\alpha} \sigma, \sigma_{\alpha \beta}:=\nabla_{\alpha} \nabla_{\beta} \sigma$.

Conclusions:

$$
\begin{aligned}
& d \sigma \stackrel{\circ}{=} 0, \quad \cdot d^{2} \sigma \stackrel{\circ}{=} g, \quad d \sigma^{\alpha} \stackrel{\circ}{=} d x^{\alpha}, \\
& d^{p}=0 \text { for } p \geqq 3, \quad d^{p} \sigma^{\alpha} \doteq 0 \quad \text { for } p \geqq 2 \text {. }
\end{aligned}
$$

Trace version of Ledger's formula:

for $p \geqq 2$.

$$
-2(p+1) d^{p} \mu \stackrel{\circ}{=}(p-1) d^{p-2} \operatorname{Ric}+\sum_{q=2}^{p-2}\left(\begin{array}{l}
p \\
q
\end{array}\right) d^{q} \sigma_{\alpha \beta} d^{p-q} \sigma^{\alpha \beta}
$$

Conclusions:

$$
\begin{aligned}
& \mu \stackrel{\circ}{=}, \quad d \mu \stackrel{\circ}{=}, \quad-3 d^{2} \mu \stackrel{\circ}{=} \text { Ric, }
\end{aligned}
$$

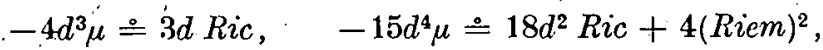

$$
\begin{aligned}
& -3 \Delta \mu \doteq R, \quad-2 d \Delta \mu \stackrel{\circ}{=} d R \text {, } \\
& -15 \Delta^{2} \mu \stackrel{\circ}{=} 12 \Delta R+2\left(\mid \text { Riem }\left.\right|^{2}-|\dot{R i c}|^{2}\right) .
\end{aligned}
$$

Special version of Ledger's formula for $n=2$ :

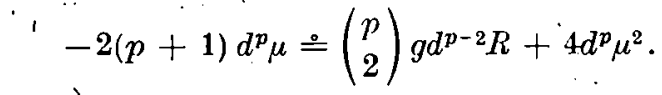

-We proceed with some technical preparations.

Proposition 1.3: For a regular two-poini function $f=f(x, y)$

$$
e \dot{\Delta}\left(|\sigma|^{l} f\right)=2|\sigma|^{l-1} D_{l} f
$$

with the linear differential expression of second order

$$
D_{l} f:=l(l+m+\mu) f+l \sigma^{\alpha} \nabla_{a} f+\frac{1}{2} \tilde{\sigma} \Delta f \text {. }
$$

Proof: Insert $f_{1}=|\sigma|^{l}, f_{2}=f \cdot$ into the product rule

$$
\Delta\left(f_{1} f_{2}\right)=\left(\Delta f_{1}\right) f_{2}+f_{1} \Delta f_{2}^{\prime}+2 g^{\alpha \beta} V_{\alpha} f_{1} \nabla_{\beta} f_{2}
$$

and use $e \Delta \cdot|\sigma|^{l}=2|\sigma|^{l-1} l(l+m+\mu)$

Proposition 1.4: In the coincidence limit

$$
d^{p} D_{l} f \stackrel{\circ}{=} \sum_{r=2}^{p}\left(\begin{array}{l}
p \\
r
\end{array}\right) d^{r} \mu d^{p-r} f+l(l+m+p) \cdot d^{p} f+\frac{1}{2}\left(\begin{array}{l}
p \\
2
\end{array}\right) g d^{p-2} \Delta f \text { for } p \geqq 2
$$

and

$$
\begin{aligned}
& -d^{p} \Delta^{q} D_{l} f=s t^{-} d^{p} \Delta^{q} f+l\left({ }^{-} d^{p} \Delta^{q} \mu\right) f+\cdots \\
& \text { for } p \geqq 2, \quad s:=l+q, \quad t:=l+m+p+q,
\end{aligned}
$$

where .... indicates terms which are of a differential order in $f$ greater than 0 and less than $p+2 q$. 
The proof of (1.12) makes use of (1.6), (1.8). For the proof of (1.13) replace $p$ in (1.12) by $p+2 q$ and apply the iterated trace operator $\operatorname{tr}^{q}$ with the help of (1.1):

$$
\begin{aligned}
& \operatorname{tr}^{q} d^{p+2 q} D_{l} f=d^{p} \Delta^{q} D_{l} f+\cdots, \\
& \left.\begin{array}{c}
p+2 q \\
2
\end{array}\right)-\operatorname{tr}^{q}\left(g d^{p+2 q-2} \Delta f\right)=2 q(m+p+q)^{-} \operatorname{tr}^{q-1}\left(d^{p+2 q-2} \Delta f\right)^{\prime}+\cdots \\
& =2 q(m+p+q)^{-} d^{p} \Delta^{q} f+\cdots
\end{aligned}
$$

Here .... indicates terms which do not contribute to the expressions (1.13). The coefficient of $-d^{p} \Delta^{q} f$ becomes

$$
l(l+m+p+2 q)+q(m+p+q)=(\dot{l}+q)(l+m+p+q)=s t
$$

\section{\$2. Derivation of the necessary conditions}

Proposition 2:1: Define two-point scalar fields $f_{k}{ }^{\prime}=f_{k}{ }^{\prime}(x, y)$ recursively with respect to $k=0,1,2, \ldots$ by

Then

$$
f_{0}^{l}:=1, \quad f_{k+1}^{l}:=D_{l-k} f_{k}^{l} \text {. }
$$

$$
(e \Delta)^{k}|\sigma|^{l}=2^{k}|\sigma|^{l-k} f_{k}^{i}
$$

The proof is done by mathematical induction with respect to $k$ and by means of (1.10)

Conclusion: $\Delta^{k} \sigma^{l}=0$ if and only if $f_{k}^{l}=0$.

Examples:

$$
\begin{aligned}
& f_{1}{ }^{l}=l(l+m+\mu), \\
& f_{2}{ }^{i}=l(l-1)\left[(l+m+\mu)(l+m-1+\mu)+\sigma^{\alpha} D_{\alpha} \mu\right]+\frac{1}{2} l \sigma \Delta \mu .
\end{aligned}
$$

Proposition 2.2: There exist the limits

$$
f_{k}:=\lim _{l \rightarrow 0} f_{k}^{l} / l \quad \text { for } \quad k \geqq 1
$$

and with these there holds

$$
\Delta^{k}(\log |\sigma|)=2^{k} \sigma^{-k} f_{k} \text {. }
$$

Proof: Mathematical induction with respect to $k$ shows that the sequence of functions which is recursively defined by

$$
f_{1}:=m+\mu, \quad f_{k+1}:=D_{-k} f_{k}
$$

fulfills both (2.5) and (2.6)

Conclusion: $\Delta^{k} \log \sigma=0$ if and only if $f_{k}=0$.

Proposition 2.3: In the coincidence limit

$$
\begin{aligned}
& f_{k}^{\prime} \doteq(k !)^{2}\left(\begin{array}{l}
l \\
k
\end{array}\right)\left(\begin{array}{c}
l+\dot{m} \\
k
\end{array}\right), \\
& f_{k} \doteq_{\alpha}(-1)^{k-1} k !(k-1) !\left(\begin{array}{c}
m \\
k
\end{array}\right) .
\end{aligned}
$$


Proof: By passing to the coincidence limits the differential recursions (2.1) shrink to the algebraic recursions

$$
\begin{array}{ll}
f_{0}^{l} \doteq 1, & f_{k+1}^{l} \stackrel{\stackrel{1}{=}(l-k)(l+m-k) f_{k}{ }^{\prime},}{f_{1} \doteq m,} \quad f_{k+1} \stackrel{\circ}{=}-k(m-k) f_{k},
\end{array}
$$

and these are solved just by $(2.8),(2.9)$

For $k \geqq 1$ one can also write

$$
\begin{aligned}
& f_{k}^{i} \doteq l(l-1) \ldots(l-k+1)(l+m)(l+m-1) \ldots(l+m-k+1), \\
& f_{k} \doteq(-1)^{k}(k-1) ! m(m-1) \ldots(m-k+1) .
\end{aligned}
$$

As a direct conclusion we get the

Theorem 2.1: If $\Delta^{k} \sigma^{l}=0$ then the power exponent $l$ is an integer with $0 \leqq l \leqq k-1$ or $l+m$ is an integer with $0 \leqq l+m \leqq k-1$. If $\Delta^{k} \log \sigma=0$ then the dimension $n$ is even and $2 \leqq n \leqq 2 k$.

Proposition 2.4: In the coincidence limit

$$
\begin{aligned}
& -d^{p} \Delta^{q} f_{k+1}^{l} \stackrel{\circ}{=}(s-k)(t-k){ }^{-} d^{p} \Delta^{q} f_{k}^{l}+(l-k)\left({ }^{-} d^{p} \Delta^{q} \mu\right) f_{k}{ }^{l}+\cdots, \\
& \text { for } p, q \geqq 0, \quad s:=l+q, \quad t:=l+m+p+q,
\end{aligned}
$$

where ... indicates terms which are of a differential order in $f_{k}{ }^{\prime}$ greater than 0 and less than $p+2 q$. For $p+2 q \leqq 3$ and for $p+2 q=4$, Ric $=0$ these residual terms vanish.

Proof: Proposition 1.4 is applied to the present situation; $l$ is to be replaced by $l-k$. For $p+2 q=4$ the individual terms are to be inspected. Especially, one obtains inductively $d f_{k}{ }^{\prime}=0$ for all $k, l$.

Proposition 2.5: Define numbers $c_{k}{ }^{\prime}=c_{k}{ }^{l}(p, q)$ by

$$
c_{k}^{i}:=\sum_{r=1}^{k}[(k-r) !]^{2}\left(\begin{array}{l}
s-r \\
k-r
\end{array}\right)\left(\begin{array}{l}
t-r \\
k-r
\end{array}\right)(i-r+1) f_{r-1}^{t} .
$$

(Take the coincidence limit of $f_{r-1}^{l}$ on the right-hand side.) Then for $1 \leqq p+2 q \leqq 3$ and for $p+2 q=4$, Ric $=0$

$$
-d^{p} \Delta^{q} f_{k}^{l} \stackrel{\circ}{=} c_{k}^{l}(p, q)-d^{p} \Delta^{q} \mu
$$

Proof: By ignoring the residual terms ... the system (2.12) becomes an algebraic recursion system for the coincidence limits. This is reduced by the ansatz (2.14) to the recursion system for the $c_{k}^{l}$

$$
c_{k+1}^{l} \stackrel{\circ}{=}(s-k)(t-k) c_{k}^{l}+(l-k) f_{k}^{l}, \quad c_{0}^{l}=0
$$

and the latter is solved just by (2.13)

Conclusion: If $\Delta^{k} \sigma^{l}=0$ then

$$
\begin{aligned}
& c_{k}{ }^{l}(0,1) R=0, \quad c_{k}{ }^{l}(2,0)-R i c=0, \\
& c_{k}{ }^{l}(1,1) d R=0, \quad c_{k}{ }^{l}(3,0)-d R i c=0 .
\end{aligned}
$$

If $\Delta^{k} \sigma^{l}=0$ and Ric $=0$ then

$$
c_{k}{ }^{l}(0,2)|R i e m|^{2}=0, \quad c_{k}{ }^{l}(4,0)^{-}(\text {Riem })^{2}=0 .
$$


Let us classify - for given $n, k, p, q-$ the values of $l$ with $f_{k}^{l} \doteq 0$ :

(i) $q \leqq k-1$ and $l$ is an integer with $0 \leqq l \leqq k-q-1$.

(ii) $q \geqq 1$ and $l$ is a positive integer with $k-q \leqq l \leqq k-1$.

(iii) $1 \leqq p+q \leqq k-1$ and $l+m$ is an integer with $0 \leqq l+m \leqq k-p-q-1$.

(iv) $1 \leqq p+q \leqq k$ and $l+m$ is a positive integer with $k-p-q \leqq l+m$ $\leqq k-1$.

Proposition 2.6: There holds

$c_{k}^{\prime l}(p, q)=0$ in the cases (i), (iii),

$c_{k} !(p, q)>0$ in the case (ii),

- $c_{k}{ }^{\prime}(p, q) \sim \sum_{r=1}^{k}\left(\begin{array}{c}s-r \\ q\end{array}\right)\left(\begin{array}{c}t-r \\ p+q-1\end{array}\right)$ in the case (iv).

If $n$ is odd or $l<0$ then the proportionality factor is non-zero here.

Proof: (i): By assumption $s \doteq l+q<k$ and thus $\left(\begin{array}{l}s-r \\ k-r\end{array}\right)=0$ for $1 \leqq r \leqq k$.

(iii): By assumption $t=l+m+p+q<k$ and thus $\left(\begin{array}{l}t-r \\ k-r\end{array}\right)=0$ for $1 \leqq r \leqq k$.

(ii): By assumption $l<k$ and because of $(l-r+1) l_{r-1}^{l} \doteq 0$ for $r>l$ the summation in (2.13) stops at $r=l$ and each of the remaining summands is positive as a product of five positive factors. (iv): By assumption $c_{k}{ }^{l}$ can be transformed into

$$
\begin{aligned}
c_{k}{ }^{\prime}= & k !(k-1) !\left(\begin{array}{l}
k \\
q
\end{array}\right)^{-1}\left(\begin{array}{c}
k-1 \\
p+q-1
\end{array}\right)^{-1}\left(\begin{array}{c}
l \\
k-q
\end{array}\right)\left(\begin{array}{c}
l+m \\
k-p-q
\end{array}\right) \\
& \times \sum_{r=1}^{k}\left(\begin{array}{c}
s-r \\
q
\end{array}\right)\left(\begin{array}{c}
t-r \\
p+q-1
\end{array}\right) .
\end{aligned}
$$
- If $l$ is half-integer or negative then $\left(\begin{array}{c}l \\ k-q\end{array}\right) \neq 0$. The other factors in front of the
sum are non-zero

Propósition' 2.7: Define numbers $c_{k}=c_{k}(p, q)$ by

$$
c_{k}=[(k-1) !]^{2} \sum_{r=1}^{k}\left(\begin{array}{l}
k-1 \\
r-1
\end{array}\right)^{-2}\left(\begin{array}{l}
q-r \\
k-r
\end{array}\right)\left(\begin{array}{l}
t-r \\
k-r
\end{array}\right)(-1)^{r-1} \cdot\left(\begin{array}{c}
m \\
r-1
\end{array}\right) \text {. }
$$

with $t:=m+p+q$. Then for $1 \leqq p+2 q \leqq 3$ and for $p+2 q=4 ;$ Ric $=0$

$$
-d^{p} \Delta^{q} f_{k} \stackrel{\circ}{=} c_{k}(p, q)-d^{p} \Delta^{q} \mu \text {. }
$$

Proof: Pass to the limit $l \rightarrow 0$ in Proposition 2.5 according to (2.5). The recursion system for the $c_{k}=c_{k}(p, q)$ reads $c_{k+1} \stackrel{\circ}{=}(q-h)(t-k) c_{k}-k f_{k}, c_{1}=1$, and is solved just by (2.17)

Conclusion: If $\Delta^{k} \log \sigma=0$ then

$$
\begin{aligned}
& c_{k}(0,1) R=0, \quad c_{k}(2,0)^{\prime} R i c=0, \\
& c_{k}(1,1) d R=0, \quad c_{k}(3,0)-d R i c=0 .
\end{aligned}
$$

If $\Delta^{k} \log \sigma=0$ and $R i c=0$ then

$$
c_{k}(0,2)|R i e m|^{2}=0, \quad c_{k}(4,0)-(\text { Riem })^{2}=0 .
$$


Proposition 2.8: If $n \geqq 3$ and $q+1 \leqq k \leqq m+p+q$ then $c_{k}(p, q) \neq 0$.

Proof: By assumption $c_{k}=c_{k}(p, q)$ can be transformed into

$$
c_{k}=(-1)^{k-1}[(k-1) !]_{r=q+1}^{2} \sum_{r}^{k}\left(\begin{array}{l}
k-1 \\
r-1
\end{array}\right)^{-2}\left(\begin{array}{c}
k-q-1 \\
k-r
\end{array}\right)\left(\begin{array}{l}
t-r \\
k-r
\end{array}\right)\left(\begin{array}{c}
m- \\
r-1
\end{array}\right)
$$

'Here all summands are positive

\section{§ 3. Results for $\boldsymbol{l}+m=\boldsymbol{k}-1$. Elomentary solutions.of the Laplace equation}

Tet us remind the notion of an elementary solution following $[9,11]$. We denote by $N$ a neighbourhood of the diagonal in $M \times M$ in which Synge's function $\sigma=\sigma(x, y)$ is defined and

$$
N^{-}:=\{(x, y) \in N \mid \dot{\mid} \sigma(x, y) \neq 0\} \text {. }
$$

Definition 3.1: Let $n \geqq 3$. A two:point function $u=u(x, y)$ which is defined and $C^{\infty}$ in a set $N^{-}$and satisfies

$$
\begin{aligned}
& \Delta u=0 \text { in } N^{*}, \\
& \lim _{x \rightarrow y}|\sigma|^{m} u=\text { const. } \neq 0
\end{aligned}
$$

is called an elementary solution of the Laplace equation.

For even $n \geqq 4$ such an elementary solution has the form

$$
u=\sigma^{-m} u+\mathfrak{u} \log |\sigma|
$$

with $C^{\infty}$ functions $\mathcal{U}=\mathcal{U}(x, y), \mathfrak{u}=\mathfrak{u}(x, y)$. The rare situations in which the logarithmic part $\mathfrak{U} \log |\sigma|$ is missing are especially interesting.

Definition 3.2 ; Let $n \geqq 4$ be even and let the two-point function $u=u(x, y)$ be defined and $C^{\infty}$ in a set $N$. An elementary solution of the Laplace equation of the form

$$
u=u(x, y)=\sigma^{-m} u(x, y) \text {. in } N^{-}
$$

is called logarithm-free.

For a lorentzian metric $g$ there exists an interpretation in other terms: The Laplace equation then admits a logarithm-free elementary solution if and only if Huygens' principle in the sense of $[9,5,12]$ is valid.

Theorem 3.1: Let

$$
\Delta^{k}|\sigma|^{k-m-1} \doteq 0
$$

and for even $n$ additionally $k \leqq m$. Then

$$
u:=\Delta^{k-1}|\sigma|^{k-m-1}
$$

is an elementary solution of the Laplace equation and for even $n$ it is logarithm-free.

Proof: The Propositions 2.1 and 2.3 in fact realize the wanted formulas (3.4) and (3.2):

$$
\begin{gathered}
\Delta^{k-1}|\sigma|^{k-m-1}=(2 e)^{k-1}|\sigma|^{-m} f_{k-1}^{k-m-1}, \\
f_{k-1}^{k-m-1} \doteq(-1)^{k-1}(k-1) !^{2}\left(\begin{array}{c}
m-1 \\
k-1
\end{array}\right)
\end{gathered}
$$


Theorem 3.2: Let $n \geqq 4$ be even and ,

$$
\Delta^{m+1}(\log |\sigma|)=0 \text {. }
$$

Then

$$
u:=\Delta^{m}(\log |\sigma|)
$$

is a logarithm-free elementary. solution of the Laplace equation.

Proof: The Propositions $2.2^{\circ}$ and 2.3 in fact realize the wanted formulas (3.4) and (3.2):

$$
\Delta^{m}(\log |\sigma|)=2^{m} \sigma^{-m} f_{m}, .{ }^{\prime} f_{m} \doteq(-1)^{m-1} m !(m-1) !
$$

Proposition 3.1: From the assumption of Theorem 3.1 there follows

$$
(n-k-1) R=0 \text { for } k \geqq 1, \quad-R i c=0 \text { for } k \geqq 2 \text {. }
$$

From the assumption of Theorem 3.1 and $R$ ic $=0$ there follows

$$
-^{-}(\text {Riem })^{2}=0 \text { for } k \geqq 4 \text {. }
$$

Proof: For $1 \leqq p+q \leqq k$ we have the case (iv) of Proposition 2.6 with a nonzero proportionality factor. Especially we get

$$
\begin{aligned}
& c_{k}^{l}(0,1) \sim 2 \sum_{r=1}^{k}(s-r)=k(k-n+1) \text { for } k \geqq 1, \\
& c_{k}^{l}(2,0) \sim \sum_{r=1}^{k}(t-r)=\left(\begin{array}{c}
t \\
2
\end{array}\right)=\left(\begin{array}{c}
k+1 \\
2
\end{array}\right) \text { for } k \geqq 2, \\
& c_{k}^{l}(4,0) \sim \sum_{r=1}^{k}\left(\begin{array}{c}
t-r \\
3
\end{array}\right)=\left(\begin{array}{c}
t \\
4
\end{array}\right)=\cdot\left(\begin{array}{c}
k+3 \\
4
\end{array}\right) \text { for } k \geqq 4,
\end{aligned}
$$

and we apply now the conclusion following Proposition 2.5

Theorem 3.3: If $\Delta^{k} \sigma^{k-m-1} \doteq 0$ and if $g$ is lorentzian and $4 \leqq k \leqq \dot{m}$ for even $n$ or $4 \leqq k \neq n-1$ for odd $n$ respectively then $(M, g)$ is flat.

The proof is composed by the Propositions 3.1 and 1.2 .

Proposition 3.2: From the assumption of Theorem 3.2 there follows

$$
\operatorname{Ric}^{\prime}=0, \quad \therefore-(\text { Riem })^{2}=0, \quad \mid \text { Riem }\left.\right|^{2}=0 .
$$

Proof: For $m \geqq q$ and $p+q \geqq 1$ we have the situation of Proposition 2.8. For the special case $n=4, p=0, q=2$ we calculate directly $c_{2}(0,2)=1$. The assertion follows now from the conclusion to Proposition 2.7

Theorem 3.4: If $\Delta^{m+1} \log \sigma=0$ and if $g$ is definite or lorentzian and $n \geqq 4$ is even then $(M, g)$ is flat.

The proof is composed by the Propositions 3.2 and 1.2

Let us now specialize $k=1$. This is only for the sake of completeness - the wellknown simply harmonic manifolds will emerge [11]. A simply harmónic manifold $(M, g)$ is characterized by

$$
\mu=0 \text { for any } n \geqq 2,: \Delta \sigma^{-m}=0 \text { for } n \geqq 3, \Delta \log \sigma=0 \text { for } n=2 \text {. }
$$


Theorem 3.5: If $\Delta \sigma^{l}=0$ with $l \neq 0$ then $l=-m$ and $(M ; g)$ is a simply harmonic manifold of a dimension $n \geqq 3$. If $\Delta \log \sigma=0$ then $(M, g)$ is a simply harmonic manifold of dimension $n=2$.

The proof follows immediately from

$$
\Delta \sigma^{l}=2 \sigma^{l-1} l(l+m+\mu), \quad \Delta \log \sigma=2 \sigma^{-1}(m+\mu)^{\cdot}
$$

Let us now specialize $k=2$.

Proposition 3.3: If $\Delta^{2} \sigma^{1-m}=0$ and $m(m-1) \neq 0$ then

$$
\begin{aligned}
& (n-3) R=0, \quad{ }^{\prime}-R i c=0, \\
& -(\text { Riem })^{2}=0, \quad n(n-1) \mid \text { Riem }\left.\right|^{2}=2 R^{2} .
\end{aligned}
$$

Proof: While (3.12) is a specialization of (3.9), we derive (3.13) more directly from (with a non-zero proportionality factor)

$$
0=d^{4} f_{2}^{1-m} \stackrel{\circ}{\sim} m\left[6\left(d^{2} \mu\right)^{2}+5 d^{4} \mu\right]-3 \dot{g} d^{2} \Delta \mu
$$

'Theorem 3.6: If $\Delta^{2} \sigma^{1-m}=0$ and $n \geqq 5$ and if $g$ is definite or lorentzian then $(M, g)$ is flat.

The proof is composed by the Propositions 3.3 and 1.2. (For definite $g$ the result is already known $[3,7]$ ),

\section{$\$ 4$. Results for other situations}

We will omit the proofs in the following as long as they run along the same lines as in $\S 3$. then

Proposition 4.1: If $\Delta^{k} \sigma^{k-m-2}=0$ and $k \geqq 2$ and for even $n$ additionally $k \leqq m+1$

$$
-R i c=0 .
$$

If $\Delta^{k} \sigma^{k-m-2}=0$ and $R=0$ and $k \geqq 4$ and for even $n$ additionally $k \leqq m+1$ then

$$
{ }^{-}(\text {Riem })^{2}=0 \text {. }
$$

Theorem 4.1: If $\Delta^{k} \sigma^{k-m-2} \doteq 0$ and if $g$ is lorentzian and $R=0$ and $k \geqq 4$ and for even $n$ additionally $k \leqq m+1$ then $(M, g)$ is flat.

Proposition 4.2: If $\Lambda^{k} \sigma^{k-m-3}=0$ and $R i c=0$ and $k \geqq 4$ and for even $n$ additionally $k \leqq m+2$ then (4.2) holds true.

Proposition 4.3: If $\Delta^{k} \sigma^{k-m-4}=0$ and $R i c=0$ and $k \geqq 4$ and for even $n$ additionally $k \leqq m+3$ then (4.2) holds true.

Proposition 4.4: If $\Delta^{k} \sigma^{k-1}=0$ and $k \geqq 2$ then $R=0$. If $\Delta^{k} \sigma^{k-1}=0$ and $k \geqq 2$ and - Ric $=0$ then $\mid$ Riem $\left.\right|^{2}=0$.

Theorem 4.2: If $\Delta^{k} \sigma^{k-1}=0$ and $n=2$ and $\dot{k} \geqq 2$ then $(M, g)$ is flat.

Proposition 4.5: If $\Delta^{k} \sigma^{k-2}=0$ and $k \geqq 3$ and $R i c=0$ then $\mid$ Riem $\left.\right|^{2}=0$.

Proposition 4.6: If $\Delta^{2} \sigma^{-m}=0$ and $n \geqq 3$ then

$$
\begin{aligned}
& -R i c=0, \quad-(\text { Riem })^{2}=0, \\
& 3(n-4) \mid \text { Riem }\left.\right|^{2}=\left(5 n^{2}-2 n-12\right)|R i c|^{2} .
\end{aligned}
$$


Proof: While (4.3) follows fròm

$$
c_{2}{ }^{-m}(2, \dot{0})=m(m+1), \quad c_{2}{ }^{-m}(4,0)=3 m(m+1),
$$

we derive (4.4) more directly from (with a non-zero proportionality factor)

$$
0=d^{4} f_{2}^{-m} \sim n\left[2\left(d^{2} \mu\right)^{2}+d^{4} \mu\right]-2 g d^{2} \Delta \mu .
$$

(For definite $g$ the results (4.4) and $-R i c=0$ are already known [3])

Theorem.4.3: If $\Delta^{2} \sigma^{-m}=0$ and $n \geqq 3$ and if $g$ is lorentzian then $(M, g)$ is flat.

Proof: From the Propositions 4.6 and 1.2 there follows that $(M, g)$ is of constant curvature. Then (4.4) gives $R=0$

Proposition 4.7: If $\Delta^{2} \sigma=0$ then

$$
R=0, \quad|R i \ddot{e} m|^{2}=|R i c|^{2} .
$$

The proof follows from $\Delta^{2} \sigma=2 \Delta \mu$ and (1.8). (Again, for definite $g$ the result is known [3])

Theorem 4.4: If $\Delta^{2} \sigma=0$ and if $g$ is definite and $n \leqq 5$ or Ric $=0$ then $(M, g)$ is flat. If $\Delta^{2} \sigma=0$ and if $g$ is definite and $n=6$ then $(M, g)$ is conformally flat.

Proof: From (4.5) there follows $(n-2)|W e y l|^{2}=(n-6)|R i c|^{2}$ for $n \geqq 3$ and definiteness arguments yield the assertion

Proposition 4.8: If $\Delta^{3} \sigma=0$ then

$$
\mid \text { Riem }\left.\right|^{2}-|R i c|^{2}+6 \Delta R=0 \text {. }
$$

The proof follows from $\Delta^{3} \sigma=2 \Delta^{2} \mu$ and (1.8)

Theorem 4.5: If $\Delta^{3} \sigma=0$ and if $g$ is definite and

$$
F: \equiv 6 n(n-1) \Delta R-(n-3) R^{2} \geqq 0
$$

then $(M, g)$ satisfies $F=0$ and is

of constant curvature for $n=3$,

flat for $n=4$ and for $n=5$,

conformally flat for $n=6$.

Proof: For $n \geqq 3$ the condition (4.6) can be transformed into

$$
n(n-1)(n-2)|W e y l|^{2}+n(n-1)(6-n)|-R i c|^{2}+(n-2) F=0 \text {. }
$$

Hence from $F \geqq 0$ there follows by definiteness arguments Weyl $=0,(n-6)-\operatorname{Ric}$ $=0, F=0$

Proposition 4.9: If $\Delta^{k} \log \sigma=0$ then

$$
\begin{aligned}
& m R=0 \text { for } 2 \leqq k \leqq m+1, \quad-R i c=0 \text { for } k \leqq m+2, \\
& m \cdot d R=0 \text { for } 2 \leqq k \leqq m+2, \quad-d \text { Ric }=0 \text { for } k \leqq m+3 .
\end{aligned}
$$

If $\dot{\Delta}^{k} \log \sigma=0$ and $R \dot{b c}=0$ then

$$
\mid \text { Riem }\left.\right|^{2}=0 \text { for } 3 \leqq k \leqq m+2, \quad-(\text { Riem })^{2}=0 \text { for } k \leqq m+4 \text {. }
$$

Theorem 4.6: If $\Delta^{k} \log \sigma=0$ and $3 \leqq k \leqq m+2$ and if $g$ is definite then $(M, g)$ is flat. If $\Delta^{k} \log \sigma=0$ and $2 \leqq k \leqq m+1$ and if $g$ is lorentzian then $(M, g)$ is flat. 
Theorem 4.7: If $\Delta^{2} \log \sigma=0$ and $n=2$ then $(M, g)$ is flat.

Proof: We evaluate (with non-zero proportionality factors)

$$
\begin{aligned}
& 0=d^{4} f_{2} \stackrel{\circ}{\sim}\left(d^{2} \mu\right)^{2}+d^{4} \mu-g d^{2} \Delta \mu, \\
& 0=d^{5} f_{2} \sim 4\left(5 d^{2} \mu d^{3} \mu+d^{5} \mu\right)-5 g d^{3} \Delta \mu
\end{aligned}
$$

with the help of (1.9). The resulting equation system

$$
9 \Delta R+7 R^{2}=0, \quad 3 d \Delta R+5 R d R=0
$$

has $R=0$ as the unique solution

\section{$\$ 5$. Non-flat manifolds for which a power of the radius is $\boldsymbol{k}$-harmonic}

A simply harmonic manifold with one of the additional properties,

$$
\begin{aligned}
& n \leqq 3 \\
& g \text { is definite or lorentzian, } \\
& g \text { is conformally flat }
\end{aligned}
$$

is known to be flat [11]. Otherwise, for each dimension $n \geqq 4$ and each signature of $g$ different from the definite or the lorentzian one there exist non-flat simply harmonic manifolds [11]. These provide exainiples for our problems.

Theorem 5.1: A simply harmonic manifold $(M, g)$ fulfills for any positive integer $k$

$$
\Delta^{k} \sigma^{k-1}=0, \quad \Delta^{k} \sigma^{k-m-1}=0 .
$$

$A$ simply harmonic manifold $(M, g)$ of even dimension $n=2 m+2$ fulfills

$$
\Delta^{m+1} \log \sigma=0 \text {. }
$$

Proof: From $\mu=0$ there follows

$$
\begin{aligned}
& \Delta^{k} \sigma^{l}=2^{k} \sigma^{l-k}(k !)^{2}\left(\begin{array}{l}
l \\
k
\end{array}\right)\left(\begin{array}{c}
l+m \\
k
\end{array}\right), \\
& \Delta^{k} \log \sigma=2^{k} \sigma^{-k}(-1)^{k-1} k !(k-1) !\left(\begin{array}{c}
m \\
k
\end{array}\right) .
\end{aligned}
$$

and this gives the assertion

Theorem 5.2: To any dimension $n \geqq 3$ and any signature of $g$ different from the definite one there exist non-flat manifolds $(M, g)$ which satisfy

$$
\Delta^{2} \sigma=0 \text {. }
$$

Proof: The metrics of the form

$$
g=2 d x^{1} d x^{2}+g_{i j}\left(x^{1}\right) d x^{i} d x^{j} \quad(i, j=3,4, \ldots, n)
$$

are generalizations of the plane gravitational waves and have the following properties:

$\Delta \sigma$ depends only on $x^{1}$ and not on the $x^{i}$,

$\Delta f\left(x^{1}\right)=0$ for any smooth function $f$,

$\operatorname{sign}\left(g_{\alpha \beta}\right)=\left(+,-, \operatorname{sign}\left(\dot{g}_{i j}\right)\right)$.

These observations - which are essentially due to [8] - yield the assertion 
Proposition 5.1: A 3-dimensional manifold of constant curvature $K$-satisfies. $\Delta_{,}^{2} \sigma=-8 K$.

The proof follows from the formulas

$$
\begin{aligned}
& \mu=-1+(2 K \sigma)^{1 / 2} \cot (2 K \sigma)^{1 / 2}=: f(\sigma), \\
& \Delta^{2} \sigma=2 \Delta \mu=4 \sigma f^{\prime \prime}(\sigma)+4(\mu+m+1) f^{\prime}(\sigma) .
\end{aligned}
$$

(For definite $g$ the result is known [2,3] and with its help in [3] higher-dimensional non-flat manifolds satisfying $\Delta^{2} \sigma=0$ are, constructed) 1 -

Theorem 5.3: The condition $\Delta^{2} \sigma^{1 / 2}=0$ characterizes 3-dimensional manifolds 'of constant curvature.

Proof: From $\Delta^{2} \sigma^{1 / 2}=0$ there follows $n=3$ by Theorem 2.1 and $-R i c=0$ by Proposition 3.3. Conversely, a calculation based on (5.4) and (2.4) shows that for a 3-dimensional manifold of constant curvature there holds $f_{2}^{1 / 2}=0$. (Again, the result is already known for definite $g[2,3,7]$ )

\section{REFERENCES}

[1] Ber, L.: Introduction d'un tenseur du quatrieme order. Compt. Rend. Acad. Sc. Paris 248 (1959), 1297-1300.

[2] Caddeo, R.: Riemannian manifolds on which the distance function is biharmonic. Rend. Sem. Mat. Univers. Politecn. 'Torino $40(1982), 93 \rightarrow 101$.

[3] Caddeo, R., and P. Matzeu: Riemannian manifolds with $\Delta^{2} r^{k}=0$. Preprint, Universitá di Cagliari 1983.

[4] CaDDEo, R., and L. VANHeCKe: Does $\Delta^{2} d^{2-n}=0$ on a Riemannian manifold imply flatness? Preprint, Kath. Universiteit Leuven 1983.

[5] Courant, R., und D. HInerrt: Methoden der mathematischen Physik. Berlin: SpringerVerlag 1968.

[6] Erchrorn, J.: Lokale Geometrie des Radius in Riemannschen Mannigfältigkeiten I. Beitr. z. Alg. u. Geom. 18 (1984), $41-48$.

[7] Eicheors, J.: Lokale Geometrie des Radius in Riemannschen Mannigfaltigkeiten II. Beitr.z. Alg. u. Geom. 18 (1984), 177-1S9.

[8] Güмtнer, P.: Ein Beispiel einer nichttrivialen Huygensschen Differentialgleichung mit vier unabhängigen Veränderlichen. Arch. Rational Mech. Anal. 18 (1965), 103-106..

[9] Hadauard, J.: Lectures on Cauchy's problem in linear partial differential equations. New Haven: Yale University Press 1923.

[10] Lichnerowicz, A., et A. G. Walker: Sur les espaces riemanniens harmoniques de type hyperbolique normal. Compt. Rend. Acid. Sc. Paris 221'(1945), 394-396.

[11] Ruse;, H. S., WaLker, A. G., and T. J. WILlmore: Harmonic Spaces. Roma: Edizioni Cremonese 1961.

[12] Scúlmming, R.: Das Huygens̈sche Prinzip bei linearen hyperbolischen Differentialgleichungen zweiter Ordnung für allgemeine Felder. Beiträge z. Analysis 11. (1978), 45-90.

[13] Synaf, J. L.: Relativity. The general theory. Amsterdam: North-Holland Publ. Comp. 1960.

Manuskripteingang: 13.03:1984; in revidierter Fassung: 15. 06.1984

VERFASSER :

Doz. Dr. sc. Ratner Schimingo

Sektion Mathematik der Ernst-Moritz-Arndt-Universität ,

DDR - 2200 Greifswald, Friedrich-Ludwig-Jahn-Str. 15 a 